\title{
Products of cyclic fuzzy finite state machines
}

\author{
Feidan Huang ${ }^{1, a^{*}}$, Zexi Deng ${ }^{1, b}$ \\ ${ }^{1}$ Department of Mathematics, Guizhou University of Engineering Science, Bijie, China 551700 \\ ahfd2001@163.com, bexideng@qq.com
}

Keywords: fuzzy finite state machines; cyclic; product; strong cyclic

Abstract. A cyclic fuzzy finite state machine is a fuzzy finite state machine which is generated by a state. In this paper, we study products of cyclic fuzzy finite state machine. We prove that two cyclic finite state machines are cyclic when the full direct product(or restricted direct product, or cascade product, or wreath product) of them is cyclic. However, products of two cyclic fuzzy finite state machines are not necessarily cyclic. We introduce the concept of strong cyclic fuzzy finite state machine, and prove that products(including full direct product, restricted direct product, cascade product and wreath product ) of two strong cyclic fuzzy finite state machines are strong cyclic.

\section{Introduction}

Wee [1] first proposed the mathematical formulation of a fuzzy automaton in 1967. Malik et al. [3-6] introduced the concepts of fuzzy finite state automata, fuzzy transformation semigroup, covering, direct product, cascade product, wreath product and Cartesian composition. Almost achievements of fuzzy automata which are obtained by Malik et al. were written in [7]. On the basis of Malik et al.'s results, fuzzy automata have been further researched[8-15]. Cyclic fuzzy finite state machine which was introduced firstly by Malik et al.[6] is a class of important fuzzy finite state machine. A primary submachine[7] of a fuzzy finite state machine is a special cyclic fuzzy finite state machine. It had been proved that every fuzzy finite state machine is an union of some primary submachines of it[7]. Malik et al. had also proved that the Cartesian composition of two fuzzy finite state machines is cyclic if and only if the two fuzzy finite state machines are cyclic[6]. In this paper, we study other products of cyclic fuzzy finite state machines.

\section{Preliminaries}

Definition 2.1 ${ }^{[5]}$ A fuzzy finite state machine (ffsm) is a triple $M=(Q, X, \mu)$, where $Q$ and $X$ are finite nonempty sets and $\mu$ is a fuzzy subset of $Q \times X \times Q$, i.e. $\mu: Q \times X \times Q \rightarrow[0,1]$.

Let $X^{*}$ denote the set of all words of elements of $X$ of finite length. The empty word is denoted by $\Lambda$, and $|u|$ denote the length of a word $u \in X^{*}$. Let $X^{+}=X^{*} \backslash\{\Lambda\}$.

Definition 2.2 $2^{[5]}$ Let $M=(Q, X, \mu)$ be a ffsm. Define the fuzzy subset $\mu^{*}$ of $Q \times X^{*} \times Q$ by:

$$
\begin{gathered}
\mu^{*}(q, \Lambda, p)=\left\{\begin{array}{l}
1, q=p \\
0, q \neq p
\end{array},\right. \\
\mu^{*}(q, x a, p)=\underset{r \in Q}{\vee}\left\{\mu^{*}(q, x, r) \wedge \mu^{*}(r, a, p)\right\}, \forall x \in X^{*}, a \in X .
\end{gathered}
$$

Definition 2.3 ${ }^{[6]}$ Let $M=(Q, X, \mu)$ be a ffsm, $q_{0} \in Q$. If $\forall q \in Q, \exists u \in X^{*}$ such that $\mu^{*}\left(q_{0}, x, q\right)>0$, then $q_{0}$ is called a generator of $M$, and $M$ is called a cyclic ffsm. If $M$ is generated by $q_{0}$, then $M$ is denoted by $M=<q_{0}>$.

Definition 2.4 Let $M=(Q, X, \mu)$ be a ffsm and $M=<q_{0}>$. If $\forall q \in Q$ and $\forall u \in X^{+}, \mu^{*}\left(q_{0}, u, q\right)>0$, then $M$ is called a strong cyclic ffsm.

Definition 2.5 ${ }^{[5]}$ Let $M_{i}=\left(Q_{i}, X_{i}, \mu_{i}\right)$ be a ffsm, $i=1,2$. Let $\eta$ be a function of $Q_{2}$ onto $Q_{1}$ and let $\xi$ be a function of $X_{1}$ into $X_{2}$. Extend $\xi$ to a function $\xi^{*}$ of $X_{1}^{*}$ into $X_{2}^{*}$ by $\xi^{*}(\Lambda)=\Lambda$ and 
$\forall u=x_{1} x_{2} \mathrm{~L} x_{n} \in X_{1}^{*}, \xi^{*}(u)=\xi\left(x_{1}\right) \xi\left(x_{2}\right) \mathrm{L} \xi\left(x_{n}\right)$. Then $(\eta, \xi)$ is called a covering of $M_{1}$ by $M_{2}$, written $M_{1} \leq M_{2}$, if and only if $\forall q_{2} \in Q_{2}, q_{1} \in Q_{1}$, and $u \in X_{1}^{*}$,

$$
\mu_{1}\left(\eta\left(q_{2}\right), u, q_{1}\right)=\underset{\eta\left(r_{2}\right)=q_{1}}{\vee} \mu_{2}\left(q_{2}, \xi^{*}(u), r_{2}\right) .
$$

Definition 2.6 ${ }^{[5]}$ Let $M_{i}=\left(Q_{i}, X_{i}, \mu_{i}\right)$ be a ffsm, $i=1,2 . M_{1} \times M_{2}=\left(Q_{1} \times Q_{2}, X_{1} \times X_{2}, \mu_{1} \times \mu_{2}\right)$ is called the full direct product of $M_{1}$ and $M_{2}$. Where

$$
\mu_{1} \times \mu_{2}\left(\left(q_{1}, q_{2}\right),\left(x_{1}, x_{2}\right),\left(p_{1}, p_{2}\right)\right)=\mu_{1}\left(q_{1}, x_{1}, p_{1}\right) \wedge \mu_{2}\left(q_{2}, x_{2}, p_{2}\right)
$$

$\forall\left(q_{1}, q_{2}\right),\left(p_{1}, p_{2}\right) \in Q_{1} \times Q_{2},\left(x_{1}, x_{2}\right) \in X_{1} \times X_{2}$.

For $u=\left(a_{1}, b_{1}\right)\left(a_{2}, b_{2}\right) \mathrm{L}\left(a_{n}, b_{n}\right) \in\left(X_{1} \times X_{2}\right)^{*}$, denote $u=\left(u_{1}, u_{2}\right)$, where $u_{1}=a_{1} a_{2} \mathrm{~L} a_{n}$ and $u_{2}=b_{1} b_{2} \mathrm{~L} b_{n}$.

Definition 2.7 ${ }^{[5]}$ Let $M_{i}=\left(Q_{i}, X, \mu_{i}\right)$ be a ffsm, $i=1,2 . M_{1} \wedge M_{2}=\left(Q_{1} \times Q_{2}, X, \mu_{1} \wedge \mu_{2}\right)$ is called the restricted direct product of $M_{1}$ and $M_{2}$. Where

$$
\mu_{1} \wedge \mu_{2}\left(\left(q_{1}, q_{2}\right), x,\left(p_{1}, p_{2}\right)\right)=\mu_{1}\left(q_{1}, x, p_{1}\right) \wedge \mu_{2}\left(q_{2}, x, p_{2}\right)
$$

$\forall\left(q_{1}, q_{2}\right),\left(p_{1}, p_{2}\right) \in Q_{1} \times Q_{2}, \forall x \in X$.

Definition 2.8 ${ }^{[5]}$ Let $M_{i}=\left(Q_{i}, X_{i}, \mu_{i}\right)$ be a ffsm, $i=1,2$. Let $\omega: Q_{2} \times X_{2} \rightarrow X_{1}$ be a function. $M_{1} \omega M_{2}=\left(Q_{1} \times Q_{2}, X_{2}, \mu^{\omega}\right)$ is called the cascade product of $M_{1}$ and $M_{2}$. Where

$$
\mu^{\omega}\left(\left(q_{1}, q_{2}\right), x,\left(p_{1}, p_{2}\right)\right)=\mu_{1}\left(q_{1}, \omega\left(q_{2}, x\right), p_{1}\right) \wedge \mu_{2}\left(q_{2}, x, p_{2}\right)
$$

$\forall\left(q_{1}, q_{2}\right),\left(p_{1}, p_{2}\right) \in Q_{1} \times Q_{2}, \forall x \in X_{2}$.

Definition 2.9 ${ }^{[5]}$ Let $M_{i}=\left(Q_{i}, X_{i}, \mu_{i}\right)$ be a ffsm, $i=1,2$. Let $f$ be a function from $Q_{2}$ into $X_{1} \cdot M_{1} \circ M_{2}=\left(Q_{1} \times Q_{2}, X_{1}^{Q_{2}} \times X_{2}, \mu^{\circ}\right)$ is called the wreath product of $M_{1}$ and $M_{2}$. Where

$$
\mu^{\circ}\left(\left(q_{1}, q_{2}\right),(f, x),\left(p_{1}, p_{2}\right)\right)=\mu_{1}\left(q_{1}, f\left(q_{2}\right), p_{1}\right) \wedge \mu_{2}\left(q_{2}, x, p_{2}\right)
$$

$\forall\left(q_{1}, q_{2}\right),\left(p_{1}, p_{2}\right) \in Q_{1} \times Q_{2}, \forall(f, x) \in X_{1}^{Q_{2}} \times X_{2}$.

Lemma 2.1 ${ }^{[5]}$ Let $M_{i}=\left(Q_{i}, X_{i}, \mu_{i}\right)$ be a ffsm, $i=1,2 . M_{1} \times M_{2}=\left(Q_{1} \times Q_{2}, X_{1} \times X_{2}, \mu_{1} \times \mu_{2}\right)$ is the full direct product of $M_{1}$ and $M_{2}$, then $\forall\left(q_{1}, q_{2}\right),\left(p_{1}, p_{2}\right) \in Q_{1} \times Q_{2}$ and $\forall\left(u_{1}, u_{2}\right) \in\left(X_{1} \times X_{2}\right)^{*}$,

$$
\left(\mu_{1} \times \mu_{2}\right)^{*}\left(\left(q_{1}, q_{2}\right),\left(u_{1}, u_{2}\right),\left(p_{1}, p_{2}\right)\right)=\mu_{1}^{*}\left(q_{1}, u_{1}, p_{1}\right) \wedge \mu_{2}^{*}\left(q_{2}, u_{2}, p_{2}\right) .
$$

Lemma 2.2 ${ }^{[5]}$ Let $M_{i}=\left(Q_{i}, X, \mu_{i}\right)$ be a ffsm, $i=1,2 . M_{1} \wedge M_{2}=\left(Q_{1} \times Q_{2}, X, \mu_{1} \wedge \mu_{2}\right)$ is the restricted direct product of $M_{1}$ and $M_{2}$, then $\forall\left(q_{1}, q_{2}\right),\left(p_{1}, p_{2}\right) \in Q_{1} \times Q_{2}$ and $\forall u \in X^{*}$,

$$
\left(\mu_{1} \wedge \mu_{2}\right)^{*}\left(\left(q_{1}, q_{2}\right), u,\left(p_{1}, p_{2}\right)\right)=\mu_{1}^{*}\left(q_{1}, u, p_{1}\right) \wedge \mu_{2}^{*}\left(q_{2}, u, p_{2}\right) .
$$

Lemma 2.3 ${ }^{[5]}$ Let $M_{i}=\left(Q_{i}, X_{i}, \mu_{i}\right)$ be a ffsm, $i=1,2 . M_{1} \omega M_{2}=\left(Q_{1} \times Q_{2}, X_{2}, \mu^{\omega}\right)$ is the cascade product of $M_{1}$ and $M_{2}$. Then $\forall\left(q_{1}, q_{2}\right),\left(p_{1}, p_{2}\right) \in Q_{1} \times Q_{2}, \forall u=a_{1} a_{2} \mathrm{~L} a_{n} \in X_{2}^{*}$ where $a_{1}, a_{2}, \mathrm{~L}, a_{n} \in X_{2}$,

$$
\mu^{\omega *}\left(\left(q_{1}, q_{2}\right), u,\left(p_{1}, p_{2}\right)\right)=
$$

$\vee\left\{\mu_{1}^{*}\left(q_{1}, \omega\left(q_{2}, a_{1}\right) \omega\left(q_{2}^{(1)}, a_{2}\right) \mathrm{L} \omega\left(q_{2}^{(n-1)}, a_{n}\right), p_{1}\right) \wedge \mu_{2}\left(q_{2}, a_{1}, q_{2}{ }^{(1)}\right) \wedge \mathrm{L} \wedge \mu_{2}\left(q_{2}^{(n-1)}, a_{n}, p_{2}\right) \mid q_{2}^{(1)}, \mathrm{L}, \in q_{2}^{(n-1)} \in Q_{2}\right\}$.

Lemma 2.4 ${ }^{[5]}$ Let $M_{i}=\left(Q_{i}, X_{i}, \mu_{i}\right)$ be a ffsm, $i=1,2 . M_{1}$ o $M_{2}=\left(Q_{1} \times Q_{2}, X_{1}^{Q_{2}} \times X_{2}, \mu^{\circ}\right)$ is the wreath product of $M_{1}$ and $M_{2}$. Then $\forall\left(q_{1}, q_{2}\right),\left(p_{1}, p_{2}\right) \in Q_{1} \times Q_{2}$, and $\forall\left(f_{1}, a_{1}\right)\left(f_{2}, a_{2}\right) \mathrm{L}\left(f_{n}, a_{n}\right) \in\left(X_{1}^{Q_{2}} \times X_{2}\right)^{*}$,

$$
\mu^{\circ *}\left(\left(q_{1}, q_{2}\right),\left(f_{1}, a_{1}\right)\left(f_{2}, a_{2}\right) \mathrm{L}\left(f_{n}, a_{n}\right),\left(p_{1}, p_{2}\right)\right)=
$$

$\vee\left\{\mu_{1}^{*}\left(q_{1}, f_{1}\left(q_{2}\right) f_{2}\left(q_{2}^{(1)}\right) \mathrm{L} f_{n}\left(q_{2}^{(n-1)}\right), p_{1}\right) \wedge \mu_{2}\left(q_{2}, a_{1}, q_{2}^{(1)}\right) \wedge \mathrm{L} \wedge \mu_{2}\left(q_{2}{ }^{(n-1)}, a_{n}, p_{2}\right) \mid q_{2}^{(i)} \in Q_{2}, i=1,2, \mathrm{~L}, n-1\right\}$

\section{Main results}

Theorem 3.1 Let $M_{i}=\left(Q_{i}, X_{i}, \mu_{i}\right)$ be a ffsm, $i=1,2$ and let $(\eta, \xi)$ be a covering of $M_{1}$ by $M_{2}$. If $M_{2}$ is cyclic and $\xi$ is surjective, then $M_{1}$ is cyclic.

Proof. Let $M_{2}=<p_{0}>$. Since $\eta$ surjective, $\forall q_{1} \in Q_{1}, \exists q_{2} \in Q_{2}$ such that $\eta\left(q_{2}\right)=q_{1}$. Since $M_{2}=<p_{0}>$, there exists $u_{2} \in X_{2}^{*}$ such that $\mu_{2}\left(p_{0}, u_{2}, q_{2}\right)>0$. Since $\xi$ is surjective, there exists $u_{1} \in X_{1}^{*}$ such that $\xi^{*}\left(u_{1}\right)=u_{2}$. By $M_{1} \leq M_{2}$,

$$
\mu_{1}\left(\eta\left(p_{0}\right), u_{1}, q_{1}\right)=\underset{\eta\left(r_{2}\right)=q_{1}}{\vee} \mu_{2}\left(p_{0}, \xi^{*}\left(u_{1}\right), r_{2}\right)=\underset{\eta\left(r_{2}\right)=q_{1}}{\vee} \mu_{2}\left(p_{0}, u_{2}, r_{2}\right) \geq \mu_{2}\left(p_{0}, u_{2}, q_{2}\right)>0 .
$$


Thus $M_{1}$ is generated by $\eta\left(p_{0}\right)$, i.e. $M_{1}$ is cyclic.

Theorem 3.2 Let $M_{i}=\left(Q_{i}, X_{i}, \mu_{i}\right)$ be a ffsm, $i=1,2$. let $M_{1} \times M_{2}=\left(Q_{1} \times Q_{2}, X_{1} \times X_{2}, \mu_{1} \times \mu_{2}\right)$ be the full direct product of $M_{1}$ and $M_{2}$. If $M_{1} \times M_{2}$ is cyclic, then $M_{1}$ and $M_{2}$ are cyclic.

Proof. Let $M_{1} \times M_{2}=<\left(q_{0}, p_{0}\right)>$. Then $\forall q_{1} \in Q_{1}$ and $\forall q_{2} \in Q_{2}, \exists\left(u_{1}, u_{2}\right) \in\left(X_{1} \times X_{2}\right)^{*}$ such that $\left(\mu_{1} \times \mu_{2}\right)^{*}\left(\left(q_{0}, p_{0}\right),\left(u_{1}, u_{2}\right),\left(q_{1}, q_{2}\right)\right)>0$.

Thus $\mu_{1}{ }^{*}\left(q_{0}, u_{1}, q_{1}\right) \wedge \mu_{2}{ }^{*}\left(p_{0}, u_{2}, q_{2}\right)>0$, and then $\mu_{1}{ }^{*}\left(q_{0}, u_{1}, q_{1}\right)>0$ and $\mu_{2}{ }^{*}\left(p_{0}, u_{2}, q_{2}\right)>0$. Hence $M_{1}=<q_{0}>$ and $M_{2}=<p_{0}>$, i.e. $M_{1}$ and $M_{2}$ are cyclic.

Remark3.1 $M_{1} \times M_{2}$ is not necessarily cyclic when $M_{1}$ and $M_{2}$ are cyclic.

Example 3.1 Let $M_{1}=\left(Q_{1}, X_{1}, \mu_{1}\right)$, where $Q_{1}=\left\{q_{1}, p_{1}\right\}, X_{1}=\{a\}$ and

$$
\mu_{1}\left(q_{1}, a, q_{1}\right)=0, \mu_{1}\left(q_{1}, a, p_{1}\right)=0.1, \mu_{1}\left(p_{1}, a, q_{1}\right)=0.2, \mu_{1}\left(p_{1}, a, p_{1}\right)=0 .
$$

Let $M_{2}=\left(Q_{2}, X_{2}, \mu_{2}\right)$, where $Q_{2}=\left\{q_{2}, p_{2}\right\}, X_{2}=\{b\}$ and

$$
\mu_{2}\left(q_{2}, b, q_{2}\right)=0, \mu_{2}\left(q_{2}, b, p_{2}\right)=0.2, \mu_{2}\left(p_{2}, b, q_{2}\right)=0.3, \mu_{2}\left(p_{2}, b, p_{2}\right)=0 .
$$

Then $M_{1} \times M_{2}=\left(Q_{1} \times Q_{2}, X_{1} \times X_{2}, \mu_{1} \times \mu_{2}\right)$, where $Q_{1} \times Q_{2}=\left\{\left(q_{1}, q_{2}\right),\left(q_{1}, p_{2}\right),\left(p_{1}, q_{2}\right),\left(p_{1}, p_{2}\right)\right\}, X_{1} \times X_{2}=\{(a, b)\}$ and

$$
\begin{aligned}
& \mu_{1} \times \mu_{2}\left(\left(q_{1}, q_{2}\right),(a, b),\left(q_{1}, q_{2}\right)\right)=0, \mu_{1} \times \mu_{2}\left(\left(q_{1}, p_{2}\right),(a, b),\left(q_{1}, p_{2}\right)\right)=0, \\
& \mu_{1} \times \mu_{2}\left(\left(p_{1}, q_{2}\right),(a, b),\left(p_{1}, q_{2}\right)\right)=0, \mu_{1} \times \mu_{2}\left(\left(p_{1}, p_{2}\right),(a, b),\left(p_{1}, p_{2}\right)\right)=0, \\
& \mu_{1} \times \mu_{2}\left(\left(q_{1}, q_{2}\right),(a, b),\left(q_{1}, p_{2}\right)\right)=0, \mu_{1} \times \mu_{2}\left(\left(q_{1}, p_{2}\right),(a, b),\left(q_{1}, q_{2}\right)\right)=0, \\
& \mu_{1} \times \mu_{2}\left(\left(q_{1}, q_{2}\right),(a, b),\left(p_{1}, q_{2}\right)\right)=0, \mu_{1} \times \mu_{2}\left(\left(p_{1}, q_{2}\right),(a, b),\left(q_{1}, q_{2}\right)\right)=0, \\
& \mu_{1} \times \mu_{2}\left(\left(p_{1}, q_{2}\right),(a, b),\left(p_{1}, p_{2}\right)\right)=0, \mu_{1} \times \mu_{2}\left(\left(p_{1}, p_{2}\right),(a, b),\left(p_{1}, q_{2}\right)\right)=0, \\
& \mu_{1} \times \mu_{2}\left(\left(p_{1}, p_{2}\right),(a, b),\left(q_{1}, p_{2}\right)\right)=0, \mu_{1} \times \mu_{2}\left(\left(q_{1}, p_{2}\right),(a, b),\left(p_{1}, p_{2}\right)\right)=0, \\
& \mu_{1} \times \mu_{2}\left(\left(q_{1}, q_{2}\right),(a, b),\left(p_{1}, p_{2}\right)\right)=0.1, \mu_{1} \times \mu_{2}\left(\left(p_{1}, p_{2}\right),(a, b),\left(q_{1}, q_{2}\right)\right)=0.2, \\
& \mu_{1} \times \mu_{2}\left(\left(q_{1}, p_{2}\right),(a, b),\left(p_{1}, q_{2}\right)\right)=0.1, \mu_{1} \times \mu_{2}\left(\left(p_{1}, q_{2}\right),(a, b),\left(q_{1}, p_{2}\right)\right)=0.2 .
\end{aligned}
$$

$M_{1}=<q_{1}>=<p_{1}>$ and $M_{2}=<q_{2}>=<p_{2}>$ are cyclic, but $M_{1} \times M_{2}$ is not cyclic.

Theorem 3.3 Let $M_{i}=\left(Q_{i}, X_{i}, \mu_{i}\right)$ be a ffsm, $i=1,2$. Let $M_{1}=<q_{0}>$ and $M_{2}=<p_{0}>$. If $M_{1}$ and $M_{2}$ are strong cyclic, then $M_{1} \times M_{2}$ is strong cyclic.

Proof. $\forall\left(q_{1}, q_{2}\right) \in Q_{1} \times Q_{2}, \forall\left(u_{1}, u_{2}\right) \in\left(X_{1} \times X_{2}\right)^{+}$, since $M_{1}$ and $M_{2}$ are strong cyclic, $\mu_{1}^{*}\left(q_{0}, u_{1}, q_{1}\right)>0$ and $\mu_{2}^{*}\left(p_{0}, u_{2}, q_{2}\right)>0$. Then $\mu_{1}^{*}\left(q_{0}, u_{1}, q_{1}\right) \wedge \mu_{2}^{*}\left(p_{0}, u_{2}, q_{2}\right)>0$. Thus $\left(\mu_{1} \times \mu_{2}\right)^{*}\left(\left(q_{0}, p_{0}\right),\left(u_{1}, u_{2}\right),\left(q_{1}, q_{2}\right)\right)>0$. Hence $M_{1} \times M_{2}=<\left(q_{0}, p_{0}\right)>$ and $M_{1} \times M_{2}$ is strong cyclic.

Theorem 3.4 Let $M_{i}=\left(Q_{i}, X, \mu_{i}\right)$ be a ffsm, $i=1,2$ and let $M_{1} \wedge M_{2}=\left(Q_{1} \times Q_{2}, X, \mu_{1} \wedge \mu_{2}\right)$ be the restricted direct product of $M_{1}$ and $M_{2}$. If $M_{1} \wedge M_{2}$ is cyclic , then $M_{1}$ and $M_{2}$ are cyclic.

Proof. Let $M_{1} \wedge M_{2}=<\left(q_{0}, p_{0}\right)>$. Then $\forall q_{1} \in Q_{1}$ and $\forall q_{2} \in Q_{2}, \exists u \in X^{*}$ such that

$$
\left(\mu_{1} \times \mu_{2}\right)^{*}\left(\left(q_{0}, p_{0}\right), u,\left(q_{1}, q_{2}\right)\right)>0 \text {. }
$$

Thus $\mu_{1}^{*}\left(q_{0}, u, q_{1}\right) \wedge \mu_{2}^{*}\left(p_{0}, u, q_{2}\right)>0$, and then $\mu_{1}^{*}\left(q_{0}, u_{1}, q_{1}\right)>0$ and $\mu_{2}{ }^{*}\left(p_{0}, u_{2}, q_{2}\right)>0$. Hence $M_{1}=<q_{0}>$ and $M_{2}=<p_{0}>$, i.e. $M_{1}$ and $M_{2}$ are cyclic.

Remark3.2 $M_{1} \wedge M_{2}$ is not necessarily cyclic when $M_{1}$ and $M_{2}$ are cyclic.

Example 3.2 Let $M_{1}=\left(Q_{1}, X, \mu_{1}\right), M_{2}=\left(Q_{2}, X, \mu_{2}\right)$, where $Q_{1}=\left\{q_{1}, p_{1}\right\}, Q_{2}=\left\{q_{2}, p_{2}\right\}, X=\{a\}$ and

$$
\begin{aligned}
& \mu_{1}\left(q_{1}, a, q_{1}\right)=0, \mu_{1}\left(q_{1}, a, p_{1}\right)=0.1, \mu_{1}\left(p_{1}, a, q_{1}\right)=0.2, \mu_{1}\left(p_{1}, a, p_{1}\right)=0, \\
& \mu_{2}\left(q_{2}, a, q_{2}\right)=0, \mu_{2}\left(q_{2}, a, p_{2}\right)=0.2, \mu_{2}\left(p_{2}, a, q_{2}\right)=0.3, \mu_{2}\left(p_{2}, a, p_{2}\right)=0 .
\end{aligned}
$$

Similarly to Example 3.1, $M_{1}$ and $M_{2}$ are cyclic, but $M_{1} \wedge M_{2}$ is not cyclic.

Theorem 3.5 Let $M_{i}=\left(Q_{i}, X, \mu_{i}\right)$ be a ffsm, $i=1,2$. If $M_{1}$ and $M_{2}$ are strong cyclic, then $M_{1} \wedge M_{2}$ is strong cyclic.

Proof. Let $M_{1}=<q_{0}>$ and $M_{2}=<p_{0}>. \forall\left(q_{1}, q_{2}\right) \in Q_{1} \times Q_{2}, \forall u \in X^{+}$, since $M_{1}$ and $M_{2}$ are strong cyclic, $\mu_{1}^{*}\left(q_{0}, u, q_{1}\right)>0$ and $\mu_{2}^{*}\left(p_{0}, u, q_{2}\right)>0$. Thus $\mu_{1}^{*}\left(q_{0}, u, q_{1}\right) \wedge \mu_{2}^{*}\left(p_{0}, u, q_{2}\right)>0$, and then

$$
\left(\mu_{1} \wedge \mu_{2}\right)^{*}\left(\left(q_{0}, p_{0}\right), u,\left(q_{1}, q_{2}\right)\right)>0 \text {. }
$$


Hence $M_{1} \wedge M_{2}=<\left(q_{0}, p_{0}\right)>$ and $M_{1} \wedge M_{2}$ is strong cyclic.

Theorem 3.6 Let $M_{i}=\left(Q_{i}, X_{i}, \mu_{i}\right)$ be a ffsm, $i=1,2$ and let $M_{1} \omega M_{2}=\left(Q_{1} \times Q_{2}, X_{2}, \mu^{\omega}\right)$ be the cascade product of $M_{1}$ and $M_{2}$. If $M_{1} \omega M_{2}$ is cyclic, then $M_{1}$ and $M_{2}$ are cyclic.

Proof. Let $M_{1} \omega M_{2}=<\left(q_{0}, p_{0}\right)>$. Then $\forall p_{1} \in Q_{1}$ and $\forall p_{2} \in Q_{2}, \exists u_{2} \in X_{2}^{*}$ such that $\mu^{\omega *}\left(\left(q_{0}, p_{0}\right), u_{2},\left(p_{1}, p_{2}\right)\right)>0$. Let $u_{2}=a_{1} a_{2} \mathrm{~L} a_{n} \in X_{2}^{*}$, then by Lemma 2.3 ,

$$
\begin{gathered}
\mu^{\omega *}\left(\left(q_{0}, p_{0}\right), u_{2},\left(p_{1}, p_{2}\right)\right)= \\
\vee\left\{\mu_{1}^{*}\left(q_{0}, \omega\left(p_{0}, a_{1}\right) \omega\left(q_{2}^{(1)}, a_{2}\right) \mathrm{L} \omega\left(q_{2}{ }^{(n-1)}, a_{n}\right), p_{1}\right) \wedge \mu_{2}\left(p_{0}, a_{1}, q_{2}{ }^{(1)}\right) \wedge \mathrm{L} \wedge \mu_{2}\left(q_{2}^{\left({ }^{n-1)}\right.}, a_{n}, p_{2}\right) \mid q_{2}^{(1)}, \mathrm{L}, q_{2}^{(n-1)} \in Q_{2}\right\} .
\end{gathered}
$$

Thus $\exists q_{2}^{(1)}, q_{2}{ }^{(2)}, \mathrm{L}, q_{2}{ }^{(n-1)} \in Q_{2}$ such that

and then

$$
\mu_{1}^{*}\left(q_{0}, \omega\left(p_{0}, a_{1}\right) \omega\left(q_{2}^{(1)}, a_{2}\right) \mathrm{L} \omega\left(q_{2}^{(n-1)}, a_{n}\right), p_{1}\right) \wedge \mu_{2}\left(p_{0}, a_{1}, q_{2}^{(1)}\right) \wedge \mathrm{L} \wedge \mu_{2}\left(q_{2}^{(n-1)}, a_{n}, p_{2}\right)>0,
$$

$$
\mu_{1}^{*}\left(q_{0}, \omega\left(p_{0}, a_{1}\right) \omega\left(q_{2}^{(1)}, a_{2}\right) \mathrm{L} \omega\left(q_{2}^{(n-1)}, a_{n}\right), p_{1}\right)>0, \mu_{2}\left(p_{0}, a_{1}, q_{2}^{(1)}\right) \wedge \mathrm{L} \wedge \mu_{2}\left(q_{2}^{(n-1)}, a_{n}, p_{2}\right)>0 .
$$

Hence $M_{1}=<q_{0}>$, i.e. $M_{1}$ is cyclic.

Since

$$
\mu_{2}\left(p_{0}, u, p_{2}\right) \geq \mu_{2}\left(p_{0}, a_{1}, q_{2}^{(1)}\right) \wedge \mathrm{L} \wedge \mu_{2}\left(q_{2}^{(n-1)}, a_{n}, p_{2}\right)>0,
$$

$M_{2}=<p_{0}>$, i.e. $M_{2}$ is cyclic.

Remark3.3 $M_{1} \omega M_{2}$ is not necessarily cyclic when $M_{1}$ and $M_{2}$ are cyclic.

Example 3.3 Let $M_{1}=\left(Q_{1}, X_{1}, \mu_{1}\right)$ and $M_{2}=\left(Q_{2}, X_{2}, \mu_{2}\right)$ be define as Example 3.1. Let

$$
\omega: Q_{2} \times X_{2} \rightarrow X_{1} ;\left(q_{2}, b\right) \text { a } a,\left(p_{2}, b\right) \text { a } a .
$$

Then for $M_{1} \omega M_{2}=\left(Q_{1} \times Q_{2}, X_{2}, \mu^{\omega}\right)$,

$$
\mu^{\omega}\left(\left(s_{1}, s_{2}\right), b,\left(r_{1}, r_{2}\right)\right)=\mu_{1}\left(s_{1}, \omega\left(s_{2}, b\right), r_{1}\right) \wedge \mu_{2}\left(s_{2}, b, r_{2}\right)=\mu_{1}\left(s_{1}, a, r_{1}\right) \wedge \mu_{2}\left(s_{2}, b, r_{2}\right),
$$

$\forall\left(s_{1}, s_{2}\right),\left(r_{1}, r_{2}\right) \in Q_{1} \times Q_{2}$. Thus $M_{1} \omega M_{2}$ is defined essentially the same as $M_{1} \times M_{2}$ in Example 3.1, hence $M_{1} \omega M_{2}$ is not cyclic.

Theorem 3.7 Let $M_{i}=\left(Q_{i}, X_{i}, \mu_{i}\right)$ be a ffsm, $i=1,2$. If $M_{1}$ and $M_{2}$ are strong cyclic, then $M_{1} \omega M_{2}$ is strong cyclic.

Proof. Let $M_{1}=<q_{0}>$ and $M_{2}=<p_{0}>. \forall\left(q_{1}, q_{2}\right) \in Q_{1} \times Q_{2}, \forall u_{2}=a_{1} a_{2} \mathrm{~L} a_{n} \in X_{2}^{+}$, since $M_{2}$ is strong cyclic, $\mu_{2}{ }^{*}\left(p_{0}, u_{2}, q_{2}\right)>0$. And then $\exists q_{2}{ }^{(1)}, q_{2}{ }^{(2)}, \mathrm{L}, q_{2}{ }^{(n-1)} \in Q_{2}$ such that $\mu_{2}\left(p_{0}, a_{1}, q_{2}^{(1)}\right) \wedge \mathrm{L} \wedge \mu_{2}\left(q_{2}{ }^{(n-1)}, a_{n}, p_{2}\right)>0$. Since $M_{1}$ is strong cyclic, $\mu_{1}^{*}\left(q_{0}, \omega\left(p_{0}, a_{1}\right) \omega\left(q_{2}^{(1)}, a_{2}\right) \mathrm{L} \omega\left(q_{2}{ }^{(n-1)}, a_{n}\right), p_{1}\right)>0$. Thus

$$
\mu_{1}^{*}\left(q_{0}, \omega\left(p_{0}, a_{1}\right) \omega\left(q_{2}^{(1)}, a_{2}\right) \mathrm{L} \omega\left(q_{2}^{(n-1)}, a_{n}\right), p_{1}\right) \wedge \mu_{2}\left(p_{0}, a_{1}, q_{2}^{(1)}\right) \wedge \mathrm{L} \wedge \mu_{2}\left(q_{2}^{(n-1)}, a_{n}, p_{2}\right)>0 .
$$

Then by Lemma 2.3,

$\mu^{\omega *}\left(\left(q_{0}, p_{0}\right), u_{2},\left(p_{1}, p_{2}\right)\right) \geq \mu_{1}^{*}\left(q_{0}, \omega\left(p_{0}, a_{1}\right) \omega\left(q_{2}{ }^{(1)}, a_{2}\right) \mathrm{L} \omega\left(q_{2}{ }^{(n-1)}, a_{n}\right), p_{1}\right) \wedge \mu_{2}\left(p_{0}, a_{1}, q_{2}{ }^{(1)}\right) \wedge \mathrm{L} \wedge \mu_{2}\left(q_{2}{ }^{(n-1)}, a_{n}, p_{2}\right)>0$. Hence $M_{1} \omega M_{2}=<\left(q_{0}, p_{0}\right)>$ and $M_{1} \omega M_{2}$ is strong cyclic.

Theorem 3.8 Let $M_{i}=\left(Q_{i}, X_{i}, \mu_{i}\right)$ be a ffsm, $i=1,2$ and let $M_{1}$ o $M_{2}=\left(Q_{1} \times Q_{2}, X_{1}^{Q_{2}} \times X_{2}, \mu^{\circ}\right)$ be the wreath product of $M_{1}$ and $M_{2}$. If $M_{1}$ o $M_{2}$ is cyclic, then $M_{1}$ and $M_{2}$ are cyclic.

Proof. Let $M_{1} \circ M_{2}=<\left(q_{0}, p_{0}\right)>$. Then $\forall p_{1} \in Q_{1}$ and $\forall p_{2} \in Q_{2}, \exists u \in\left(X_{1}^{Q_{2}} \times X_{2}\right)^{*}$ such that

$$
\mu^{\omega^{*}}\left(\left(q_{0}, p_{0}\right), u,\left(p_{1}, p_{2}\right)\right)>0 .
$$

Let $u=\left(f_{1}, a_{1}\right)\left(f_{2}, a_{2}\right) \mathrm{L}\left(f_{n}, a_{n}\right)$, then by Lemma 2.4,

$$
\begin{gathered}
\mu^{\circ *}\left(\left(q_{0}, p_{0}\right),\left(f_{1}, a_{1}\right)\left(f_{2}, a_{2}\right) \mathrm{L}\left(f_{n}, a_{n}\right),\left(p_{1}, p_{2}\right)\right)= \\
\vee\left\{\mu_{1}^{*}\left(q_{0}, f_{1}\left(p_{0}\right) f_{2}\left(q_{2}{ }^{(1)}\right) \mathrm{L} f_{n}\left(q_{2}{ }^{(n-1)}\right), p_{1}\right) \wedge \mu_{2}\left(p_{0}, a_{1}, q_{2}{ }^{(1)}\right) \wedge \mathrm{L} \wedge \mu_{2}\left(q_{2}{ }^{\left({ }^{2}-1\right)}, a_{n}, p_{2}\right) \mid q_{2}{ }^{(i)} \in Q_{2}, i=1,2, \mathrm{~L}, n-1\right\} .
\end{gathered}
$$

Thus $\exists q_{2}^{(1)}, q_{2}{ }^{(2)}, \mathrm{L}, q_{2}{ }^{(n-1)} \in Q_{2}$ such that

and then

$$
\mu_{1}^{*}\left(q_{0}, f_{1}\left(p_{0}\right) f_{2}\left(q_{2}{ }^{(1)}\right) \mathrm{L} f_{n}\left(q_{2}^{(n-1)}\right), p_{1}\right) \wedge \mu_{2}\left(p_{0}, a_{1}, q_{2}{ }^{(1)}\right) \wedge \mathrm{L} \wedge \mu_{2}\left(q_{2}{ }^{(n-1)}, a_{n}, p_{2}\right)>0,
$$

$$
\mu_{1}^{*}\left(q_{0}, f_{1}\left(p_{0}\right) f_{2}\left(q_{2}^{(1)}\right) \mathrm{L} f_{n}\left(q_{2}^{(n-1)}\right), p_{1}\right)>0, \mu_{2}\left(p_{0}, a_{1}, q_{2}^{(1)}\right) \wedge \mathrm{L} \wedge \mu_{2}\left(q_{2}^{(n-1)}, a_{n}, p_{2}\right)>0 .
$$

Hence $M_{1}=<q_{0}>$, i.e. $M_{1}$ is cyclic. Since

$$
\mu_{2}\left(p_{0}, a_{1} a_{2} \mathrm{~L} a_{n}, p_{2}\right) \geq \mu_{2}\left(p_{0}, a_{1}, q_{2}{ }^{(1)}\right) \wedge \mathrm{L} \wedge \mu_{2}\left(q_{2}{ }^{(n-1)}, a_{n}, p_{2}\right)>0,
$$


$M_{2}=<p_{0}>$, i.e. $M_{2}$ is cyclic.

Remark3.4 $M_{1} \circ M_{2}$ is not necessarily cyclic when $M_{1}$ and $M_{2}$ are cyclic.

Example 3.4 Let $M_{1}=\left(Q_{1}, X_{1}, \mu_{1}\right)$ and $M_{2}=\left(Q_{2}, X_{2}, \mu_{2}\right)$ be define as Example 3.1. Since

$$
\mu^{\circ}\left(\left(s_{1}, s_{2}\right),(f, b),\left(r_{1}, r_{2}\right)\right)=\mu_{1}\left(s_{1}, f\left(s_{2}\right), r_{1}\right) \wedge \mu_{2}\left(s_{2}, b, r_{2}\right)=\mu_{1}\left(s_{1}, a, r_{1}\right) \wedge \mu_{2}\left(s_{2}, b, r_{2}\right),
$$

$\forall\left(s_{1}, s_{2}\right),\left(r_{1}, r_{2}\right) \in Q_{1} \times Q_{2}$ and $\forall(f, b) \in X_{1}^{Q_{2}} \times X_{2}, M_{1} \circ M_{2}$ is defined essentially the same as $M_{1} \times M_{2}$ in

Example 3.1, hence $M_{1} \mathrm{o} M_{2}$ is not cyclic.

Theorem 3.9 Let $M_{i}=\left(Q_{i}, X_{i}, \mu_{i}\right)$ be a ffsm, $i=1,2$. If $M_{1}$ and $M_{2}$ are strong cyclic, then $M_{1} \mathrm{o} M_{2}$ is strong cyclic.

Proof. Let $M_{1}=<q_{0}>$ and $M_{2}=<p_{0}>. \forall\left(q_{1}, q_{2}\right) \in Q_{1} \times Q_{2}$ and $\forall u=\left(f_{1}, a_{1}\right)\left(f_{2}, a_{2}\right) \mathrm{L}\left(f_{n}, a_{n}\right) \in\left(X_{1}^{Q_{2}} \times X_{2}\right)^{*}$, let $u_{2}=a_{1} a_{2} \mathrm{~L} a_{n}$. Since $M_{2}$ is strong cyclic, $\mu_{2}{ }^{*}\left(p_{0}, u_{2}, q_{2}\right)>0$. And then $\exists q_{2}{ }^{(1)}, q_{2}{ }^{(2)}, \mathrm{L}, q_{2}{ }^{(n-1)} \in Q_{2}$ such that $\mu_{2}\left(p_{0}, a_{1}, q_{2}^{(1)}\right) \wedge \mathrm{L} \wedge \mu_{2}\left(q_{2}{ }^{(n-1)}, a_{n}, p_{2}\right)>0$. Since $M_{1}$ is strong cyclic, $\mu_{1}^{*}\left(q_{0}, f_{1}\left(p_{0}\right) f_{2}\left(q_{2}{ }^{(1)}\right) \mathrm{L} f_{n}\left(q_{2}{ }^{(n-1)}\right), p_{1}\right)>0$. Thus

$$
\mu_{1}^{*}\left(q_{0}, f_{1}\left(p_{0}\right) f_{2}\left(q_{2}^{(1)}\right) \mathrm{L} f_{n}\left(q_{2}^{(n-1)}\right), p_{1}\right) \wedge \mu_{2}\left(p_{0}, a_{1}, q_{2}{ }^{(1)}\right) \wedge \mathrm{L} \wedge \mu_{2}\left(q_{2}{ }^{(n-1)}, a_{n}, p_{2}\right)>0 .
$$

By Lemma 2.4,

$$
\begin{gathered}
\mu^{0 *}\left(\left(q_{0}, p_{0}\right),\left(f_{1}, a_{1}\right)\left(f_{2}, a_{2}\right) \mathrm{L}\left(f_{n}, a_{n}\right),\left(p_{1}, p_{2}\right)\right) \geq \\
\mu_{1}^{*}\left(q_{0}, f_{1}\left(p_{0}\right) f_{2}\left(q_{2}{ }^{(1)}\right) \mathrm{L} f_{n}\left(q_{2}{ }^{(n-1)}\right), p_{1}\right) \wedge \mu_{2}\left(p_{0}, a_{1}, q_{2}{ }^{(1)}\right) \wedge \mathrm{L} \wedge \mu_{2}\left(q_{2}{ }^{(n-1)}, a_{n}, p_{2}\right)>0 .
\end{gathered}
$$

Hence $M_{1} \circ M_{2}=<\left(q_{0}, p_{0}\right)>$ and $M_{1} \circ M_{2}$ is strong cyclic.

\section{Conclusion}

In this paper, we obtain the following conclusions.

(1) we prove that $M_{1}$ and $M_{2}$ are cyclic when the full direct product(or restricted direct product, or cascade product, or wreath product) of $M_{1}$ and $M_{2}$ is cyclic.

(2) we give counter examples to show that products of $M_{1}$ and $M_{2}$ are not cyclic when $M_{1}$ and $M_{2}$ are cyclic.

(3) By introducing the concept of strong cyclic fuzzy finite automata, we prove that products (full direct product, restricted direct product, cascade product and wreath product) of $M_{1}$ and $M_{2}$ are strong cyclic when $M_{1}$ and $M_{2}$ are strong cyclic.

\section{Acknowledgements}

The project is supported by the Joint Funds of Guizhou Province under the Grant NO. J Word LKB [2012]10 and NO. J Word LKB [2012]17, Open Fund of Guangxi Key Laboratory of Hybrid Computation and IC Design Analysis under the Grant NO.HCIC201404.

\section{References}

[1] W.G. Wee: On generalizations of adaptive algorithm and application of the fuzzy sets concept to pattern classification(Ph.D. Thesis, Purdue University, 1967).

[2] K. Asai, S. Kitajima: Inform. Sci. Vol. 39 (1971) , p. 343

[3] D.S. Malik, J.N. Mordeson, M.K. Sen: Fuzzy Sets and Systems Vol. 68 (1994), p. 83

[4] D.S. Malik, J.N. Mordeson, M.K. Sen: J. Fuzzy Math. Vol. 4 (1994) , p. 781

[5] D.S. Malik, J.N. Mordeson, M.K. Sen: Fuzzy Sets and Systems Vol. 92 (1997) , p. 95

[6] D.S. MalikJ.N. MordesonM.K. Sen: Kybernetes Vol. 24(4)(1995), p. 98

[7] J.N. Mordeson, D.S. Malik: Fuzzy Automata and Languages: Theory and Applications (Chapman and Hall/CRC, Boca Raton London NewYork Washington,D.C, 2002). 
[8] H.V. Kumbhojkar, S.R. Chaudhari: Fuzzy Sets and Systems Vol. 125 (2002), p. 215

[9] W.Cheng, Z.W.Mo: Fuzzy Sets and Systems Vol. 114(2004), p. 439

[10] Tatjana Petkovic: Fuzzy Sets and Systems Vol. 157(2006), p. 444

[11] D.W.Qiu: Fuzzy Sets and Systems Vol. 141(2004), p. 391

[12] Z.H. Li, P. Li, Y.M. Li: Information Sciences Vol. 176(2006), p. 2208

[13] Y.M. Li, W. Pedrycz: Fuzzy Sets and Systems Vol. 156(2005), p. 68

[14] J. Liu, Z.W. Mo, D. Qiu, Y. Wang: Fuzzy Sets and Systems Vol. 160 (2009) , p. 2401

[15] Y. M. Li, Q.Wang: Fuzzy Sets and Systems Vol. 249 (2014) , p. 27 